\title{
STM on polycrystalline thin films
}

\author{
G Reiss, Institut für Angewandte Physik III, Universität Regensburg, Regensburg, FRG
}

\begin{abstract}
Examples for correlations of results of Scanning Tunnelling Microscopy (STM) with thickness dependent physical properties of thin films will be discussed: surface roughness enhances the thickness dependent resistivity. This effect can be described quantitatively by STM imaging. Good agreement with a simple model of the film resistivity can be found for $\mathrm{Au}$ and $\mathrm{Cr}-\mathrm{Au}$ films. The magnetization loops of $\mathrm{Au}-\mathrm{Fe}-\mathrm{Au}$ films depend on the preparation parameters, although RHEED indicates identical flat surfaces. STM, however, often shows different local topographies. Again a direct correlation can be established.
\end{abstract}

\section{Introduction}

Thickness dependent thin film properties are influenced by the surface topography. Typical features being between some $\mathrm{nm}$ and some $\AA$, direct imaging was nearly impossible till the development of the STM ${ }^{1,2}$. This method, however, provides reproducible results ${ }^{3}$ with reasonable resolution ${ }^{4}$ and thus gives a chance of a direct correlation of the surface structures with thin film properties.

Two examples will be discussed: the correlation of the surface topographies with: (i) the thickness dependent resistivity of $\mathrm{Au}$ single and $\mathrm{Cr}-\mathrm{Au}$ double layer, and (ii) with the magnetization loops of $\mathrm{Au}-\mathrm{Fe}-\mathrm{Au}$ triple layer films.

For ex situ performed STM, Au surfaces have been investigated. On $\mathrm{Au}$, atomic resolution was obtained in air $^{3,5}$; surface features are thus not strongly affected by gas adsorbates.

\section{Surface topography and film resistivity}

The thickness $(d)$ dependent resistivity $\rho_{\mathrm{f}}(d)$ is usually described by averaging the local resistivity ${ }^{6,7}$ over the film area ${ }^{8,9}$. Different roughnesses must be considered: a local microroughness $h_{\rho}\left(h_{\rho} \leqslant \lambda_{\mathrm{F}} ; \lambda_{\mathrm{F}}\right.$ is the Fermi wavelength) giving rise to either specular $\left(h_{\rho}=0\right)$ or diffuse $\left(h_{\rho}=\lambda_{\mathrm{F}}\right)$ electronic surface scattering ${ }^{6,7}$ and a macroroughness $H_{\rho}\left(H_{\rho}>\lambda_{\mathrm{F}}\right)$ describing large scale fluctuations of $d$. A combination of semi-classical results of Fuchs ${ }^{6}$ with recent quantum-mechanical calculations of Tešanović et $a l^{7}$ and the model of $\mathrm{Namba}^{8}$ gives the approximation

$\rho_{\mathrm{f}}(d) \approx \rho_{\infty}\left[1-\left(\frac{H_{\rho}}{d}\right)^{2}\right]^{-1 / 2}+\frac{3}{8} \rho_{\infty} l_{\infty} \frac{h_{\rho}}{\lambda_{\mathrm{F}} d}\left[1-\left(\frac{H_{\rho}}{d}\right)^{2}\right]^{-3 / 2}$

( $l_{\infty}$ : electronic mean free path). Fitting calculations thus provide an indirect method to obtain $h_{\rho}$ and $H_{\rho}$.

Figure 1 shows in situ measured $\rho_{\mathrm{f}}$ vs $d_{\mathrm{Au}}$ curves for $\mathrm{Cr}_{d_{\mathrm{Cr}}}{ }^{-}$ $\mathrm{Au}_{d_{\mathrm{Au}}}$ double layers evaporated on glass ${ }^{3}$. The very thin $\mathrm{Cr}$ films do not contribute to the conductivity but drastically modify the growth of the Au film itself: whereas Au on glass $\left(d_{\mathrm{Cr}}=0\right)$ shows an onset of the resistivity at $d_{\mathrm{on}} \approx 10 \mathrm{~nm}, d_{\mathrm{on}}$ is reduced to about $3 \mathrm{~nm}$ by a $\mathrm{Cr}$ precoverage of only $1.5 \mathrm{~nm}$. For

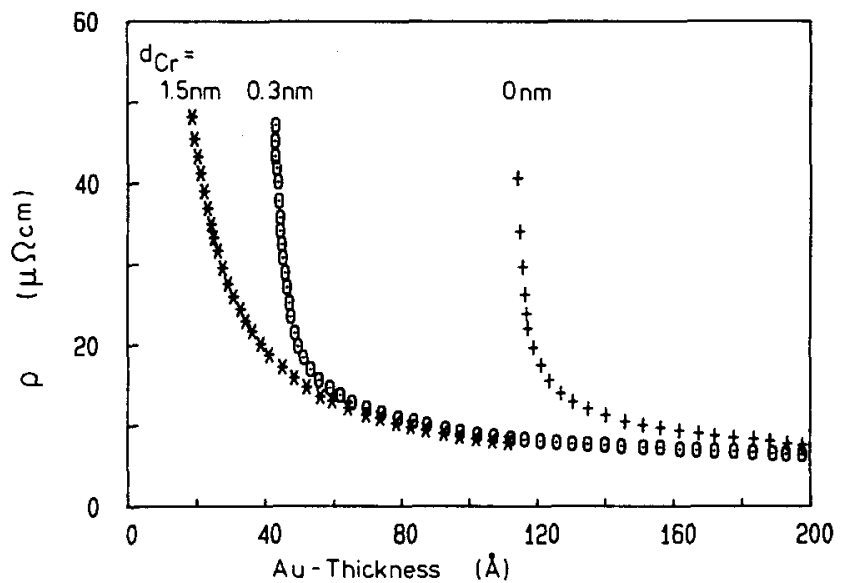

Figure 1. The resistance of $\mathrm{Cr}_{d \mathrm{C}_{r}}-\mathrm{Au}_{d d_{u}}$ double layered films on glass vs $d_{\mathrm{Au}}$. The thickness of the $\mathrm{Cr}$ precoverage is noted at each curve.

$d_{\text {on }}$ can be roughly identified with $H_{\rho}$ (equation (1)), Au films on precoated substrates thus seem to be smoother than single Au films. A quantitative analysis gives $h_{\rho}=(0.3 \pm 0.05) \mathrm{nm}$ and $H_{\rho}=(11 \pm 1) \mathrm{nm}$ for $\mathrm{Au}$ on glass; the corresponding values for $\mathrm{Cr}_{1.5 \mathrm{~nm}}-\mathrm{Au}_{d_{\mathrm{Au}}}$ are $h_{\rho}=(0.2 \pm 0.05) \mathrm{nm}$ and $H_{\rho}=(3.5 \pm 0.5) \mathrm{nm}$. These values, however, have been obtained indirectly. Direct STM imaging therefore provides a check of their reliability. Figures $2(a-c)$ show STM images of a single Au (2(a) large area, 2(b) zoom-scan) and a $\mathrm{Cr}_{1.5 \mathrm{~nm}}{ }^{-}$ $\mathrm{Au}_{30 \mathrm{~nm}}$ film on glass (2(c) $)^{3}$.

Figures 1, 2(a and c) illustrate a good correspondence between the conclusions of the $\rho_{\mathrm{f}}$ vs $d$ dependence and the measured surface topography. The single Au film (Figure 2(a)) shows a large roughness, whereas $\mathrm{Cr}-\mathrm{Au}$ is much smoother. $\mathrm{A}$ quantitative analysis yields $H_{\mathrm{STM}}=(10 \pm 2) \mathrm{nm}$ for the Au film and $H_{\mathrm{STM}}=(3 \pm 1) \mathrm{nm}$ for the $\mathrm{Cr}-\mathrm{Au}$ double layer in good agreement with the corresponding values of $H_{\rho}$. Small area STM scans (Figure 2(b)) supply information relevant for the surface scattering of electrons $\left(h_{\mathrm{STM}} \leqslant \lambda_{\mathrm{F}},{ }^{6.7}\right)$ : The single $\mathrm{Au}$ film shows a very high density of steps. Only on the top of the 


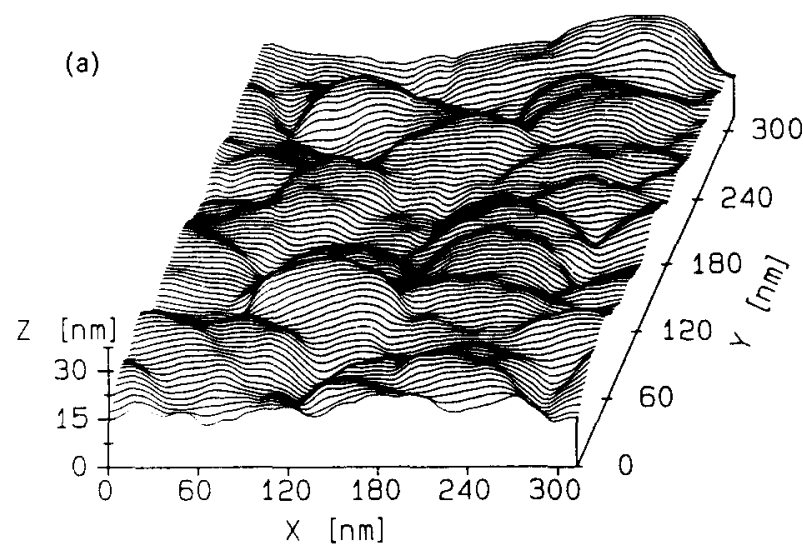

(b)
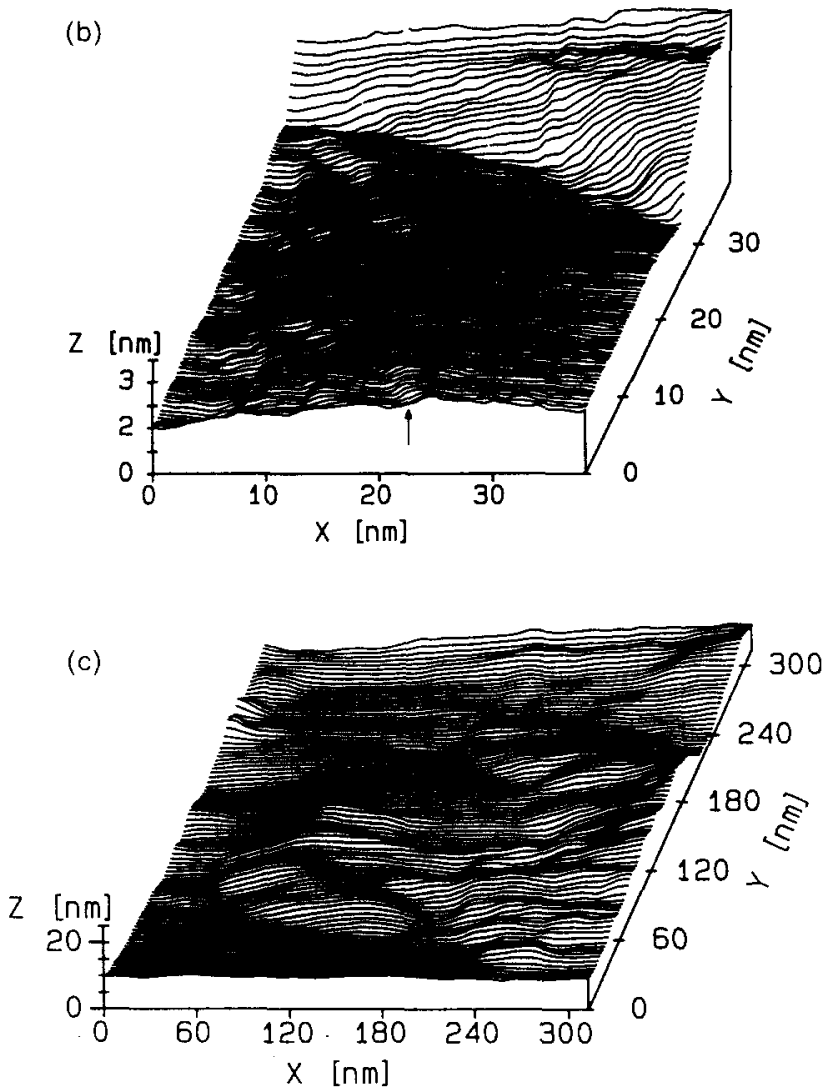

Figure 2. (a) STM topography of a $\mathrm{Au}$ film evaporated on glass, demonstrating the rough structure of these films. (b) STM image of a small area of a Au film on glass, showing flat areas separated by small steps $0.2-0.3 \mathrm{~nm}$ in height. (c) Topography of a $\mathrm{Cr}_{1.5 \mathrm{~nm}}-\mathrm{Au}_{30 \mathrm{~nm}}$ double layered thin film: a reduced roughness compared with $\mathrm{Au}$ single layers and the existence of large, atomically flat areas can be observed.

hills mainly monoatomic steps $0.2-0.3 \mathrm{~nm}$ in height, separated by more than one screening length can be observed. Average yields are $h_{\mathrm{STM}}>\lambda_{\mathrm{F}}$ at the sides and $h_{\mathrm{STM}} \approx 0.2 \mathrm{~nm}$ at the top of the hills. A rather diffuse scattering of the conduction electrons at these surfaces can thus be expected ${ }^{6.7}$. The $\mathrm{Cr}-\mathrm{Au}$ double layer (Figure 2(c)) exhibits large flat areas $\left(h_{\mathrm{STM}} \approx 0.2 \mathrm{~nm}\right)$. Enhanced specularity of this surface therefore can be expected from STM imaging in good agreement with the evidences of the $\rho_{\mathrm{f}}$ vs $d$ evaluation.

STM therefore provides new and valuable information concerning thickness dependent transport properties of thin films.
The next example discusses correlations between surface topographies and the magnetization of ferromagnetic thin films.

\section{Surface topography and magnetization}

The magnetization of thin $\mathrm{Fe}$ films sandwiched between $\mathrm{Au}$ strongly depends on the structure of the $\mathrm{Fe}$ and of the interfaces. For very thin Fe layers, the easy axis of the magnetization (ea) is usually forced parallel to the film by form anisotropies. In the case of $\mathrm{Fe}(110)$ and $\mathrm{Au}(111)$, however, an out of plane ea was reported ${ }^{10}$ for less than three $\mathrm{ml}$ of $\mathrm{Fe}$. Assuming a layer-by-layer growth, this was attributed ${ }^{10}$ to interface interactions of $\mathrm{Au}(111)$ and $\mathrm{Fe}(110)^{11}$.

A key for the interpretation is thus the knowledge of the topography of the underlying $\mathrm{Au}$ film. In order to obtain layer-by-layer growth of $\mathrm{Fe}$, atomically flat $\mathrm{Au}(111)$ substrate films must be prepared.

RHEED experiments ${ }^{10,12}$ showed flat $A u$ films for (quartz) substrate temperatures of $330 \mathrm{~K}$ and residual gas pressures $\left(p_{\mathrm{a}}\right)$ during preparation between $5 \times 10^{-9}$ and $5 \times 10^{-11} \mathrm{mbar}$, provided the substrates had been annealed at $650 \mathrm{~K}$ prior to the gold evaporation (evaporation rate $1-2 \mathrm{ml} \mathrm{s}^{-110.13}$ ). The inplane magnetization loops of $6 \mathrm{ml}$ Fe films $\left(M^{\|}, 330 \mathrm{~K}\right.$ vibrating sample magnetometer (VSM)), however, show large differences between films condensed at different $p_{\mathrm{a}}$ : the films
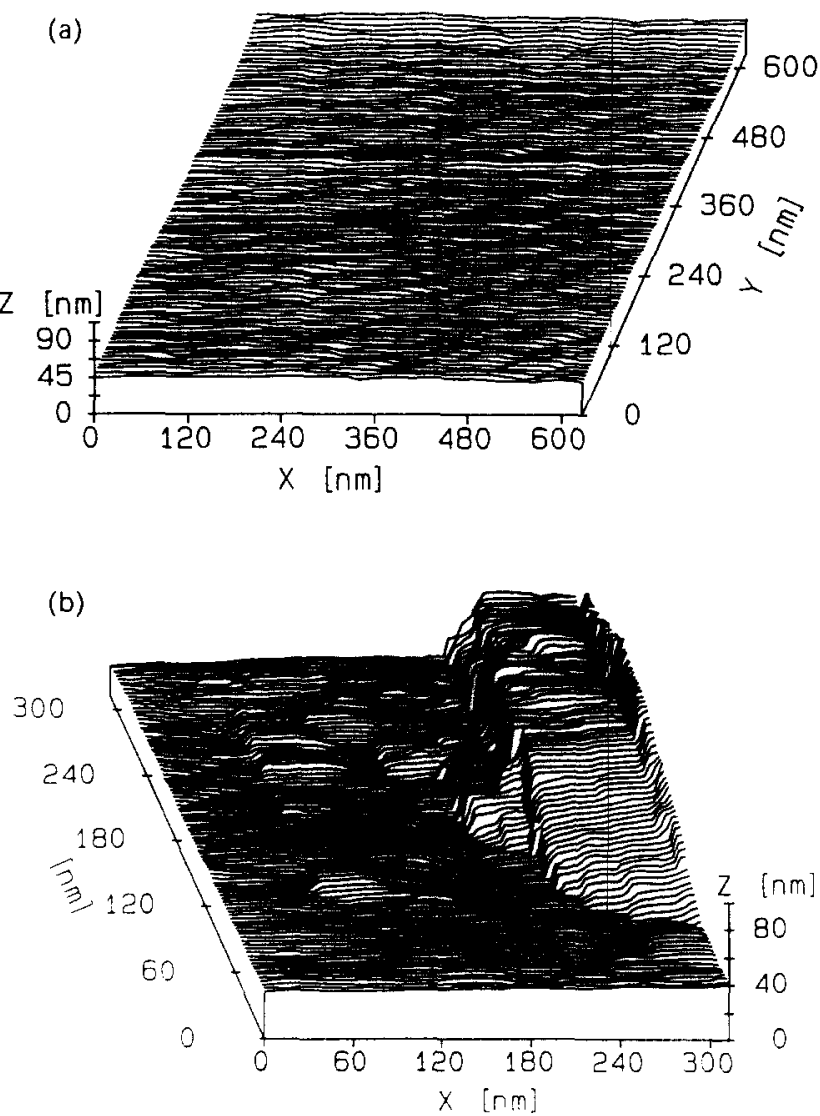

Figure 3. (a) STM topography of the underlying Au film of a $\mathrm{Au}-\mathrm{Fe}-$ Au triple layer, deposited on quartz glass at a residual gas pressure of $5 \times 10^{-11}$ mbar (other parameters: see text). (b) Surface of a $\mathrm{Au}$ substrate film evaporated under the same conditions as the film shown in Figure 3(a) except for an enhanced residual gas pressure $\left(5 \times 10^{-9} \mathrm{mbar}\right)$ 
prepared at smaller $p_{\mathrm{a}}$ exhibit a saturation magnetization $M_{\mathrm{s}}^{\|}$ $80 \%$ larger and a coercivity $H_{\mathrm{c}}^{\|} 30 \%$ smaller than those deposited under enhanced $p_{\mathrm{a}}{ }^{13}$.

This can be attributed to a reduced roughness of the Au films prepared at $5 \times 10^{-11}$ mbar compared with those evaporated at $5 \times 10^{-9}$ mbar: a rough underlying film gives rise to complex interfaces of the $\mathrm{Au}-\mathrm{Fe}-\mathrm{Au}$ sandwich. RHEED and interferometry, however, indicated flat substrate films in both cases $^{12,13}$. These methods, however, integrate over large areas. Thus STM can provide more detailed information; the results are shown in Figures 3(a and b) (3(a) $p_{\mathrm{a}}=5 \times 10^{-11} \mathrm{mbar}$, 3(b) $p_{\mathrm{a}}=5 \times 10^{-9} \mathrm{mbar}$ ).

The substrate films prepared at smaller $p_{\mathrm{a}}$ show large areas $\left(>1 \mu \mathrm{m}^{2}\right.$ ) with a residual roughness below $0.2 \mathrm{~nm}$. Fe films of more than $4 \mathrm{ml}$ on these substrates therefore exhibit small $H_{\mathrm{c}}^{\|}$ and large $M_{s}^{\|}$already at $330 \mathrm{~K}$ in agreement with the VSM measurements.

Au films grown at $5 \times 10^{-9}$ mbar only exhibit smooth surface areas of about $0.05 \mu \mathrm{m}^{2}$, separated by steps of different height $(2, \ldots, 40 \mathrm{~nm})$. Thin Fe films on these substrates therefore consist of small patches; additionally, layer-by-layer growth will be supressed by the larger sticking coefficient at the edges on the Au substrate. Compared with the Fe layers on flat Au films, a larger $H_{\mathrm{c}}$ and a reduced $M_{\mathrm{s}}^{\|}$can thus be expected from STM in agreement with the results of VSM ${ }^{10,13}$.

The crossover from ea in-plane to out-of-plane, however, could only be attributed to a interface anisotropy for flat $\mathrm{Au}$ substrates at an $\mathrm{Fe}$ thickness of about $3 \mathrm{ml}$. For rough $\mathrm{Au}-\mathrm{Fe}$ interfaces, the switching of anisotropy occurred at much larger thickness ${ }^{10,13}$.

\section{Conclusions}

STM imaging on thin films resolves surface structures larger than-roughly speaking - the shape of the tunnelling tip. Careful preparation of the tips gives the possibility to image features of vertical and lateral extensions of only some nms.

STM therefore provides valuable information concerning the influence of specific topographies on thickness dependent physical properties of thin films. The magnitude of the roughness ranges between some $\mathrm{nms}$ (comparable to the electronic $\mathrm{mfp}$ ) and some $\AA$ (comparable to the Fermi wavelength), giving rise either to geometric fluctuations of the film thickness or to modified electronic surface scattering and magnetic size effects. Even for 'quasi-epitaxial' films, STM offers additional, more detailed information than integrating methods.

Considerable further improvement will be the use of real in situ STMs, i.e. preparation and imaging under continuous uhv conditions. Moreover, force microscopy will provide detailed insight concerning electronic potentials and magnetic structures of thin film surfaces.

\section{Acknowledgements}

I am indebted to $\mathrm{Mr} \mathrm{G}$ Lugert for valuable discussions and VSM measurements, to $\mathrm{Dr} \mathrm{J}$ Vancea, $\mathrm{Mr} \mathrm{H}$ Brückl, Mr F Schneider and Mr K Bauer (STM measurements) and to $C$ Marlière and D Renard from the Institute d'Optique, Universite Paris-Sud, Orsay, for RHEED experiments. This work was supported in part by the Siemens AG.

\section{References}

' G Binning and H Rohrer, Phys Rev Lett, 49, 57 (1982).

${ }^{2} \mathrm{G}$ Binning and H Rohrer, IBM Jl Res Dev, 30, 355 (1986).

${ }^{3} \mathrm{~J}$ Vancea, G Reiss, F Schneider, K Bauer and H Hoffmann, Surface Sci, 218, 108 (1989).

${ }^{4} \mathrm{G}$ Reiss, J Vancea, H Wittmann, J Zweck and H Hoffmann, J Appl Phys, 67, 1156 (1990).

${ }^{5} \mathrm{~V}$ M Hallmark, S Chiang, J F Rabolt, J D Swalen and R J Wilson, Phys Rev Lett, 59, 2879 (1987).

${ }^{6} \mathrm{~K}$ Fuchs, Proc Camb Phil Soc, 34, 100 (1938).

${ }^{7} \mathrm{Z}$ Tešanović, M V Jarić and S Maekawa, Phys Rev Lett, 57, 2760 (1986).

${ }^{8}$ Y Namba, Japan J Appl Phys, 9, 1326 (1970).

${ }^{9} \mathrm{G}$ Reiss, K Kapfberger, G Meier, J Vancea and H Hoffmann, $J$ Phys Condens Matter, 1, 1275 (1989).

${ }^{10} \mathrm{G}$ Lugert and G Bayreuther, Thin Solid Films, 175, 311 (1989).

11 J G Gay, R Richter, J Appl Phys, 61, 3362 (1987).

${ }^{12} \mathrm{C}$ Marlière, J P Chauvineau and D Renard, Thin Solid Films, To be published.

${ }^{13} \mathrm{G}$ Lugert, $\mathrm{G}$ Reiss, $\mathrm{K}$ Bauer, $\mathrm{F}$ Schneider, $\mathrm{J}$ Vancea and $\mathrm{H}$ Hoffmann, To be published. 